\title{
Continuous Spinal Anesthetic in a Patient with Severe Aortic Stenosis for Total Knee Arthroplasty
}

\author{
Drew Jensen, MD and Adam C Young, MD* \\ Department of Anesthesiology, Rush University Medical Center, Chicago, IL, USA
}

\begin{abstract}
Patients with severe aortic stenosis represent a serious perioperative challenge for anesthesiologists when presenting for orthopedic surgery. Avoiding general anesthesia can be beneficial by averting the dramatic hemodynamic changes that can occur at induction and emergence. However, regional anesthesia can similarly produce significant alterations in sympathetic tone in blocked regions. We discuss the successful use of a continuous spinal anesthetic in a patient with severe aortic stenosis undergoing elective total knee arthroplasty.
\end{abstract}

\section{Introduction}

Patients with aortic stenosis (AS) can prove challenging to manage for anesthesiologists when presenting for elective noncardiac surgery. Reports suggest that patients with AS undergoing noncardiac surgery are at higher risk of perioperative cardiovascular events and mortality [1-3]. A stenotic aortic valve leads to left ventricular outflow obstruction and subsequent left ventricular hypertrophy; the hypertrophied ventricle increases myocardial oxygen requirements. Further complicating the matter is that oxygen delivery to myocardial tissue is impaired in patients with AS [4]. Anesthetic management focuses on avoidance of hypotension and maintenance of normal sinus rhythm, in an effort to avoid left ventricular failure secondary to coronary hypoperfusion. Because of this, neuraxial anesthetics have generally been avoided in AS patients to prevent the hypotension associated with these techniques [5]. However, though counterintuitive, a few reports suggest a carefully titrated neuraxial anesthetic may be a viable and safe alternative to general anesthesia in patients with AS undergoing noncardiac surgery $[6,7]$. We present our experience managing a patient with severe AS undergoing a total knee arthroplasty (TKA) using a continuous spinal anesthetic and intraoperative sedation. Of note, Institutional Review Board (IRB) approval was obtained and the patient gave consent for case presentation and publication.

\section{Case Discussion}

A 55-year-old male presented for a primary left TKA. He was $182 \mathrm{~cm}$ (6 feet) tall and weighed $176 \mathrm{~kg}$ (390 pounds); body mass index (BMI) was 53 . His medical history was significant for hyperlipidemia, obstructive sleep apnea, and severe AS (valve area of $0.85 \mathrm{~cm}^{2}$, aortic jet velocity of 3.59 $\mathrm{m} / \mathrm{s}$, mean valve gradient of $44 \mathrm{mmHg}$ ). His surgical history was significant for prior cervical spine fusion, limiting his neck range of motion, and an airway exam demonstrated a Mallampati class IV view. Given his habitus, history, and physical examination, a difficult intubation was anticipated. Though peripheral blocks for TKA in an AS patient has been described [8], our patient's overall body habitus made this approach particularly challenging. Following a discussion of the risks, benefits, and alternatives of general versus regional anesthesia, a continuous spinal anesthetic was chosen for this procedure. An intrathecal catheter was chosen over an epidural catheter due to their liability of bilateral, dense neuraxial block, and the ability to accurately titrate the level of blockade within a relatively short timeframe. This allowed us to anesthetize only the regions necessary to perform the proposed surgical procedure.

In the preoperative area, large bore IV access was obtained and a radial arterial line was placed. In the sitting position, identification of appropriate bony landmarks of the spine was accomplished with ultrasound. Using an $18 \mathrm{~g}$ Touhy needle, a $20 \mathrm{~g}$ closed tip catheter was introduced into the intrathecal space. Then, $0.5 \mathrm{~mL}$ of isobaric $0.5 \%$ bupivacaine was administered through the catheter. With this initial dose, hemodynamic parameters remained unchanged. After 10 minutes, a sensory exam only revealed a block of the L4S2 dermatomes and was determined to be inadequate for surgical anesthesia. An additional $0.5 \mathrm{~mL}$ of isobaric $0.5 \%$

*Corresponding author: Adam C Young, MD, Department of Anesthesiology, Rush University Medical Center, Chicago, IL, USA, Tel: 312-942-5000

Accepted: April 13, 2019

Published online: April 15, 2019

Citation: Jensen D, Young AC (2019) Continuous Spinal Anesthetic in a Patient with Severe Aortic Stenosis for Total Knee Arthroplasty. J Clin Anesth Pain Manag 3(1):51-52 
bupivacaine was administered. Following an additional 10 minutes, a sensory exam revealed a T10 sensory level bilaterally. Hemodynamic parameters again remained stable. At this point, our patient was taken to the operating room for the procedure. Intraoperative sedation was accomplished with propofol $30 \mathrm{mcg} / \mathrm{kg} / \mathrm{min}$ and dexmedetomidine 0.4 $\mathrm{mcg} / \mathrm{kg} / \mathrm{hr}$. An additional $1 \mathrm{~mL}$ of intrathecal isobaric $0.5 \%$ bupivacaine (in divided doses) was given intraoperatively to maintain a satisfactory sensory level. A total of $400 \mathrm{mcg}$ phenylephrine was administered throughout the case to maintain blood pressures near preoperative values. During the entirety of the procedure, mean arterial pressure was above $70 \mathrm{mmHg}$ and heart rate less than 70 beats per minute. Overall, the case proceeded uneventfully. Intraoperatively, our patient received $1800 \mathrm{ml}$ of intravenous crystalloid solution and he produced $600 \mathrm{ml}$ of urine. Estimated blood loss was $300 \mathrm{ml}$. The intrathecal catheter was removed at the end of the case without issue.

Sensory and motor function returned on the evening of post-operative day (POD) 0 , at which time our patient began endorsing pain at the surgical site. An adductor canal block was performed under ultrasound guidance postoperatively without complication. On POD 2, our patient was deemed stable for discharge by the surgical service, and was discharged home.

\section{Discussion}

Although considered a relative contraindication to neuraxial anesthesia, our case demonstrates that low-dose, continuous intrathecal administration of local anesthetic can be safely employed in patients with AS. Although this is not the first reported use of this technique in patients with severe AS, this report demonstrates an uncommon anesthetic approach to patients with severe AS undergoing elective lower-limb surgery and the added challenge of a known difficult airway. Judicious titration of intrathecal local anesthetic coupled with close hemodynamic monitoring allowed us to execute this technique safely for our patient and could be considered by anesthesiologists confronted with a similar scenario.

\section{Financial Disclosures}

None.

\section{Conflicts of Interest}

None.

\section{References}

1. Zahid M, Sonel AF, Saba S, et al. (2005) Preoperative risk of noncardiac surgery associated with aortic stenosis. Am J Cardiol 96: 436-438.

2. Agarwal S, Rajamanickam A, Bajaj NS, et al. (2013) Impact of aortic stenosis on postoperative outcomes after noncardiac surgeries. Circ Cardiovasc Qual Outcomes 6: 193-200.

3. Andersson C, Jorgensen ME, Martinsson A, et al. (2014) Noncardiac surgery in patients with aortic stenosis: A contemporary study on outcomes in a matched sample from the Danish health care system. Clin Cardiol 37: 680-686.

4. Samarendra P, Mangione MP (2015) Aortic stenosis and perioperative risk with noncardiac surgery. J Am Coll Cardiol 65: 295-302.

5. Frogel J, Galusca D (2010) Anesthetic considerations for patients with advanced valvular heart disease undergoing noncardiac surgery. Anesthesiol Clin 28: 67-85.

6. McDonald SB (2004) Is neuraxial blockade contraindicated in the patient with aortic stenosis? Reg Anesth Pain Med 29: 496-502.

7. Lopez MM, Guasch E, Schiraldi R, et al. (2016) Continuous spinal anaesthesia with minimally invasive haemodynamic monitoring for surgical hip repair in two patients with severe aortic stenosis. Rev Bras Anestesiol 66: 82-85.

8. Forero M, Kruisselbrink R, Leon SJ (2015) Ultrasound-guided peripheral nerve blocks for total knee arthroplasty in the context of severe aortic stenosis: Avoiding the general anesthetic. Can J Anaesth 62: 665-666.

DOI: $10.36959 / 377 / 326$

Copyright: (c) 2019 Jensen D, et al. This is an open-access article distributed under the terms of the Creative Commons Attribution License, which permits unrestricted use, distribution, and reproduction in any medium, provided the original author and source are credited. 